\title{
Precise and nondestructive characterization of a 'buried' nanostructure in a polymer thin film using synchrotron radiation ultra-small angle $\mathrm{X}$-ray scattering
}

\author{
Takamichi Shinohara $^{1,2}$, Tomoko Shirahase ${ }^{3}$, Daiki Murakami ${ }^{3,4}$, Taiki Hoshino ${ }^{3,4}$, Moriya Kikuchi ${ }^{3,4}$, \\ Jun-ichiro Koike ${ }^{5}$, Misao Horigome ${ }^{5}$, Hiroyasu Masunaga ${ }^{6}$, Hiroki Ogawa ${ }^{6}$ and Atsushi Takahara ${ }^{1,2,3,4,6}$
}

Nanoimprint lithography (NIL) is a simple and high-resolution patterning method. Nanoimprinted structures can be fabricated not only as surface structures but also as 'buried' structures for applications such as electro-optical devices. However, a precise and nondestructive evaluation method for nanoimprinted structures has not yet been established. Synchrotron radiation ultrasmall angle X-ray scattering (SR-USAXS) is a nondestructive and high-resolution characterization method. In this study, we fabricated nanostructures on a poly(lactic acid) (PLA) film using NIL. In addition, the nanoimprinted PLA film was covered with a polystyrene thin film to fabricate a 'buried' structure. The fabricated surface and the 'buried' structure were evaluated using SR-USAXS. The scattering pattern was clearly obtained from the surface and the 'buried' structure. The size of the 'buried' structure, which was estimated from the diffraction pattern, was in good agreement with that of the surface structure. These results indicate that SR-USAXS is powerful tool for the nondestructive and precise characterization of surface and 'buried' structures.

Polymer Journal (2013) 45, 307-312; doi:10.1038/pj.2012.133; published online 27 June 2012

Keywords: 'buried' structure; nanoimprint lithography; surface structure; synchrotron radiation ultra-small angle X-ray scattering

\section{INTRODUCTION}

Nanoimprint lithography ${ }^{1,2}$ (NIL) is a high-resolution surface patterning method. Molds that have various types of surface morphologies, such as lines, spaces, holes and moth-eye structures, are mechanically pressed onto a polymer substrate, which transfers the nanostructures onto the surfaces. Because NIL is simple and low cost, much research in various fields has focused on NIL, for example, for applications such as biomedical devices and electro-optical devices. ${ }^{3,4}$ Scanning electron microscopy (SEM) and atomic force microscopy have been employed for evaluating NIL patterns. However, SEM observations require pretreatment steps, such as vapor-deposition coating of conductive metals, which change the sample dimensions. Obtaining the exact morphology of nanoimprinted structures, which have markedly uneven sharp edge features, is difficult using atomic force microscopy because of the convolution of the tip shape with the surface morphological features. Furthermore, the nanoimprinted structures are not always at the outermost surface but are often utilized in 'buried' conditions for applications such as for increasing the light-emitting intensity of light-emitting diodes ${ }^{5,6}$ or increasing the power conversion efficiency of solar cells. ${ }^{7,8}$ Characterizing these 'buried' structures using microscopic techniques requires the sample to be destroyed. Therefore, precise and nondestructive evaluation methods for nanoimprinted surfaces, especially 'buried' structures, are required.

In this study, we present the application of small angle X-ray scattering (SAXS) for the structural analysis of 'buried' surfaces, which is a technique that is widely used for investigating molecular aggregation states such as crystallization behavior, ${ }^{9}$ microphase separation $^{10}$ and chain conformation in solution. ${ }^{11,12}$ SAXS is a nondestructive evaluation method that can provide structural information ranging from the $\mathrm{nm}$ to submicron scale with high resolution far beyond the optical diffraction limit. Synchrotron radiation provides a source of X-rays that have high intensity and directional characteristics. Therefore, when synchrotron radiation is used as the light source for SAXS, we can establish a long sample-todetector distance, which can be used to characterize large structures. Therefore, synchrotron radiation ultra-small X-ray scattering (SR-USAXS) is expected to be a powerful tool for the precise characterization of the periodic structure of polymer thin films. ${ }^{13,14}$ The precise characterization of the cross-sectional structure, including

${ }^{1}$ Graduate School of Engineering, Kyushu University, Fukuoka, Japan; ${ }^{2}$ International Institute for Carbon-Neutral Energy Research (WPI-I²CNER), Kyushu University, Fukuoka, Japan; ${ }^{3}$ Institute for Materials Chemistry and Engineering, Kyushu University, Fukuoka, Japan; ${ }^{4}$ Japan Science and Technology Agency (JST), ERATO Takahara Soft Interface Project, Fukuoka, Japan; ${ }^{5}$ DIC Corporation, 631 Sakado, Sakura, Chiba, Japan and ${ }^{6}$ Japan Synchrotron Radiation Research Institute (JASRI)/SPring-8, Sayo-cho, Sayo-gun, Hyogo, Japan

Correspondence: Professor A Takahara, Institute for Materials Chemistry and Engineering, Kyushu University, 744 Motooka, Nishi-ku, Fukuoka 819-0395, Japan. E-mail: takahara@cstf.kyushu-u.ac.jp

Received 8 March 2012; revised and accepted 10 May 2012; published online 27 June 2012 
the aspect ratio, line-edge roughness and structural fluctuation, could be obtained by combining SR-USAXS with X-ray reflectivity or grazing incidence SAXS. ${ }^{15,16}$

In this study, we fabricated nanostructures on the surface of a polymer (poly(lactic acid); PLA) film using NIL. PLA was chosen because it has potential applications for various biomedical devices. The fabricated structures were evaluated using microscopic observation and SR-USAXS of the outermost surface and 'buried' structures, and the ability of these techniques to characterize buried structures is discussed.

\section{EXPERIMENTAL PROCEDURE}

\section{Materials}

PLA (LACEA H280, Mitsui Chemicals, Inc., Tokyo, Japan; $M_{\mathrm{n}}=100000$, $\left.M_{\mathrm{w}} / M_{\mathrm{n}}=1.98\right)$ was supplied by Mitsui Chemicals, Inc. PLA has a glass transition temperature of $331 \mathrm{~K}$. The PLA was dissolved in chloroform and purified by precipitation into methanol. This PLA is in an amorphous state because it contains $12 \%$ D-lactide. Polystyrene (PS) was synthesized via radical polymerization. The molecular weight of PS is 92000 and its molar-mass dispersity is 2.41 .

\section{Preparation of the nanostructured film}

PLA pellets were compression molded at $453 \mathrm{~K}$ under a nominal pressure of $10 \mathrm{MPa}$ for $2 \mathrm{~min}$ to prepare the PLA film. The thickness of the PLA film was approximately $200 \mu \mathrm{m}$.

NIL was conducted using a NANOIMPRINTER NW-0401 (Meisyo Kiko Co., Ltd., Hyogo, Japan). Silicon grading mold with a line/space $=$ ca. $200 \mathrm{~nm} /$ $200 \mathrm{~nm}$ (LS 200, NTT Advanced Technology, Co, Tokyo, Japan) was used for the NIL. The mold was coated with a perfluorinated monolayer using an Optool DSX (Daikin Industries Ltd., Osaka, Japan, $0.1 \mathrm{wt} \%$ in methoxynonafluorobutane (HFE7100)) to reduce the adhesion between the mold and the film after NIL. ${ }^{4}$ A schematic diagram of the NIL process is presented in Figure 1. At first, the mold and the PLA film were heated to $353 \mathrm{~K}$, which is higher than the PLA glass transition temperature. Then, the mold was pressed onto the PLA film at $20 \mathrm{MPa}$ for $3 \mathrm{~min}$ under vacuum conditions and was removed from the PLA film after cooling to $303 \mathrm{~K}$.

To fabricate the 'buried' structure, the nanoimprinted PLA film (NIL_PLA) was layered with a PS thin film using the floating method. A glass wafer was cleaned by irradiation with ultraviolet light in a vacuum for $10 \mathrm{~min}$. The PS thin film was spin-cast from a toluene solution that contained PS ( $5 \mathrm{wt} \%$ ) onto the cleaned glass wafer at 1000 r.p.m. for 30 s. The thickness of the spin-cast PS film was determined to be ca. $600 \mathrm{~nm}$ by atomic force microscopy. The spincast PS film was peeled from the substrate and floated on water, and then the floating PS film was placed on the NIL_PLA surface (PS/NIL_PLA).

\section{Measurements}

The wetting behavior of the NIL_PLA and PS/NIL_PLA surfaces was measured using a Theta T-200 (Attention, Inc., Espoo, Finland) equipped with a video camera. A $2 \mu \mathrm{l}$ water droplet was placed onto the surfaces, and the static contact angles were recorded.

The surface morphologies of the NIL_PLA and PS/NIL_PLA films were observed using SEM (VE-7800, Keyence Co., Ltd., Osaka, Japan). For the SEM observations, the NIL_PLA and PS/NIL_PLA films were coated with osmium using a sputter coater (HPC-1SW, VACUUM DEVICE Inc., Ibaraki, Japan) to prevent the films from becoming charged and therefore damaged.

SR-USAXS was performed at the $\mathrm{BL} 03 \mathrm{XU}^{17}$ beam line of SPring-8 (Japan Synchrotron Radiation Research Institute, Hyogo, Japan) using an incident $\mathrm{X}$-ray wavelength of $0.1 \mathrm{~nm}$. The scattered X-rays were detected using a $300 \times 300 \mathrm{~mm}$ imaging plate detector, which has a pixel size of $100 \times 100 \mu \mathrm{m}$. The sample-to-detector distance was calibrated to be $7994 \mathrm{~mm}$ using the diffraction peaks of collagen. The scattering vector, $q$, is defined as $q=(4 \pi / \lambda)$ $\sin \theta$, where $\lambda$ is the wavelength of the X-rays, and $2 \theta$ is the angle between the incident X-ray beam and the scattered X-rays.

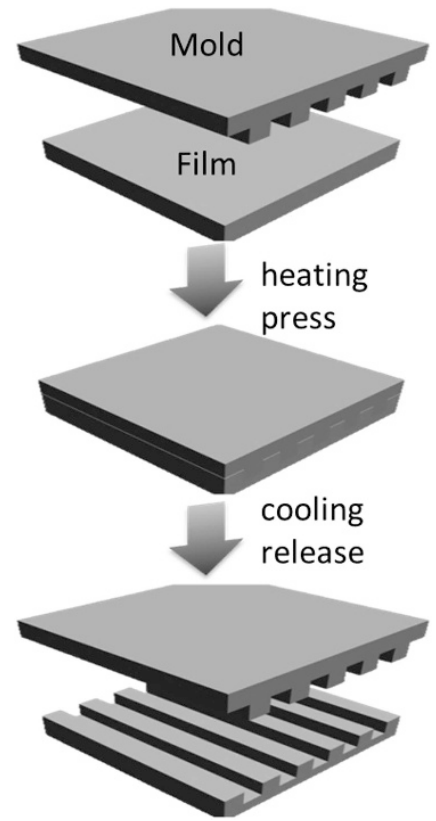

Figure 1 Schematic diagram of the NIL method. A full color version of this figure is available at Polymer Journal online.

\section{RESULTS AND DISCUSSION}

\section{SEM observation}

Figure 2 presents the SEM images of the mold and the prepared sample films. It was confirmed that the PLA film was sufficiently flat initially (Figure 2a) and that the surface nanostructure of the mold (Figure 2b) was successfully transferred onto the film (Figure 2c). However, the surface nanopattern was not observed on the PS/NIL_PLA film because the surface of the NIL_PLA film was completely covered by the PS thin film (Figure 2d). These results indicate that the 'buried' structure was successfully fabricated.

\section{Wetting behavior}

To evaluate the surface properties and the nanotexture of the fabricated PLA films, the wetting behavior was characterized. Figure 3 presents optical micrographs of a water droplet on the NIL_PLA and a schematic image of the measurement geometry. Table 1 summarizes the static contact angles of the water droplet on the PLA films. The water droplet on the NIL_PLA film was deformed along the line direction, and the static contact angle in the orthogonal direction (Figure 3b) was larger than that in the parallel direction (Figure 3c). This anisotropic wetting behavior could be explained by the pinning effect in the space occupied with air, resulting from the surface line/space (LS) pattern. ${ }^{3}$ However, an isotropic water droplet on the PS/NIL_PLA film was observed (Figures 3d and e), and the contact angle of $95^{\circ}$ was the same as that on the spin-cast PS film. This result suggests that the surface of the PS/NIL_PLA film was almost flat.

\section{Characterization of the surface and the 'buried' structure}

There are several methods for characterizing 'buried' structures. Next, the characterization of the nanostructure fabricated on the outermost side and inside of the polymer film was attempted using SR-USAXS. Figure 4 presents the two-dimensional scattering patterns of the mold and the PLA films. No scattering spot pattern is observed from a flat PLA film (Figure 4a). In contrast, anisotropic scattering patterns were 

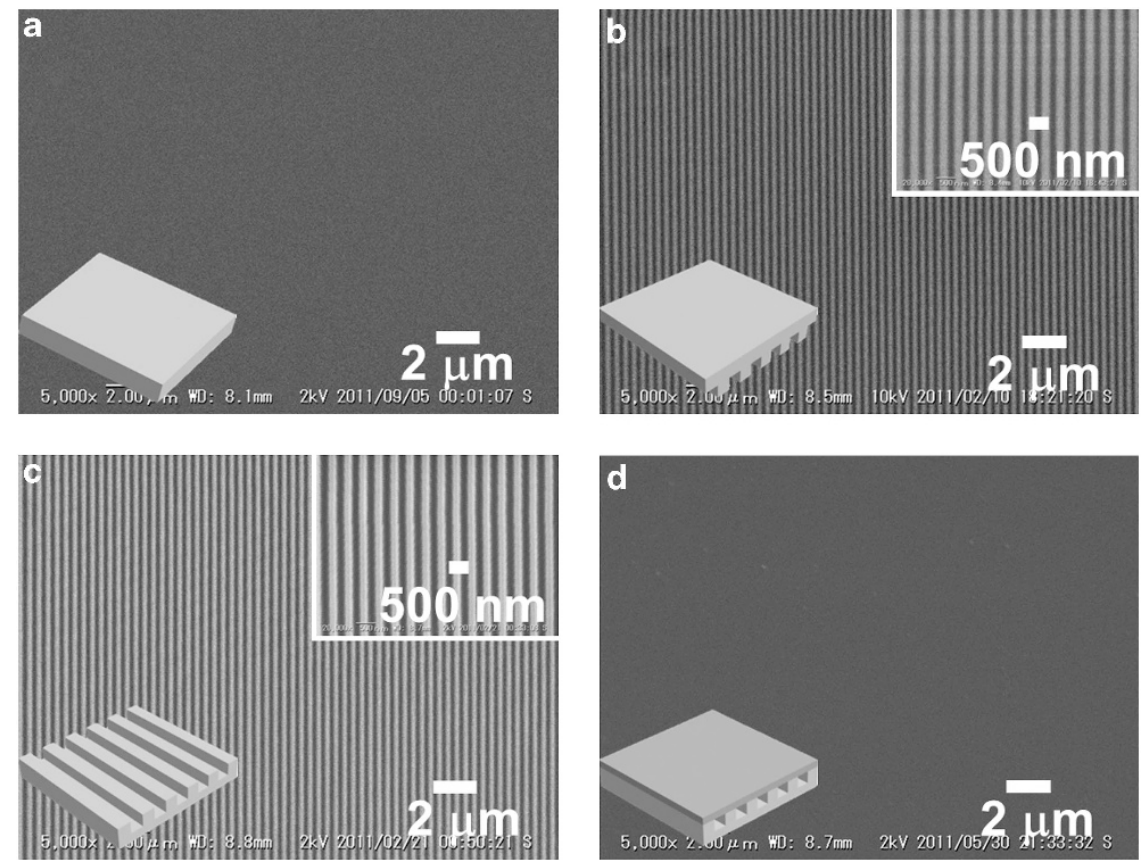

Figure 2 SEM images of the prepared samples. (a) Compression-molded PLA film, (b) the mold with a line pattern, (c) nanoimprinted PLA film (NIL_PLA) and (d) NIL_PLA film covered by a PS thin film (PS/NIL_PLA). A full color version of this figure is available at Polymer Journal online.
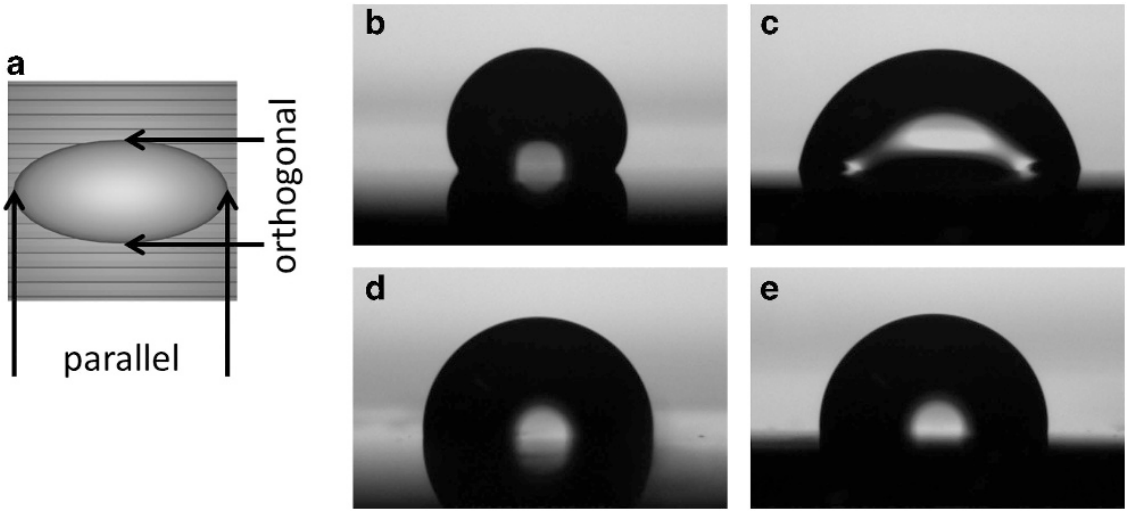

Figure 3 Optical micrograph of a water droplet on the prepared films. (a) Schematic image of the measurement geometry, (b) orthogonal direction to the line of the NIL_PLA film, (c) parallel direction to the line of the NIL_PLA film, (d) orthogonal direction to the line of the PS/NIL_PLA film and (e) parallel direction to the line of the PS/NIL_PLA film. A full color version of this figure is available at Polymer Journal online.

Table 1 Static contact angles of prepared films

\begin{tabular}{lccc}
\hline & \multicolumn{3}{c}{ Contact angle () } \\
\cline { 2 - 4 } Sample name & Arbitrarily & Parallel & Orthogonal \\
\hline Flat_PLA & $83.0 \pm 1.6$ & - & - \\
NIL_PLA & - & $71.4 \pm 0.9$ & $126.1 \pm 1.1$ \\
Flat_PS & $96.3 \pm 0.5$ & - & - \\
PS/NIL_PLA & - & $95.5 \pm 1.1$ & $95.2 \pm 1.4$ \\
\hline
\end{tabular}

clearly observed up to the 10th order from the mold and the NIL_PLA and PS/NIL_PLA films ${ }^{18}$ (Figures $4 \mathrm{~b}-\mathrm{d}$ ). These anisotropic scattering spots were attributed to the surface and the 'buried' nanostructures. In this case, the transmitted X-rays were clearly detected, even through the $0.65 \mathrm{~mm}$ thick $\mathrm{Si}$, because of the high intensity of the incident X-ray beam. Furthermore, the observation of higher-order peaks indicates that the surface nanostructure has high regularity.

The periodic sizes of the nanoimprinted structures were estimated simply, using equation (1):

$$
d=\frac{2 \pi n}{q}
$$

where $d, q$ and $n$ are the periodic size, the peak top position of the scattering X-ray and the diffraction order, respectively. The estimated pitches of the mold and the NIL_PLA and PS/NIL_PLA films are 411, 410 and $411 \mathrm{~nm}$, respectively. The good agreement of the periodic size values between the NIL_PLA and PS/NIL_PLA films indicates that 

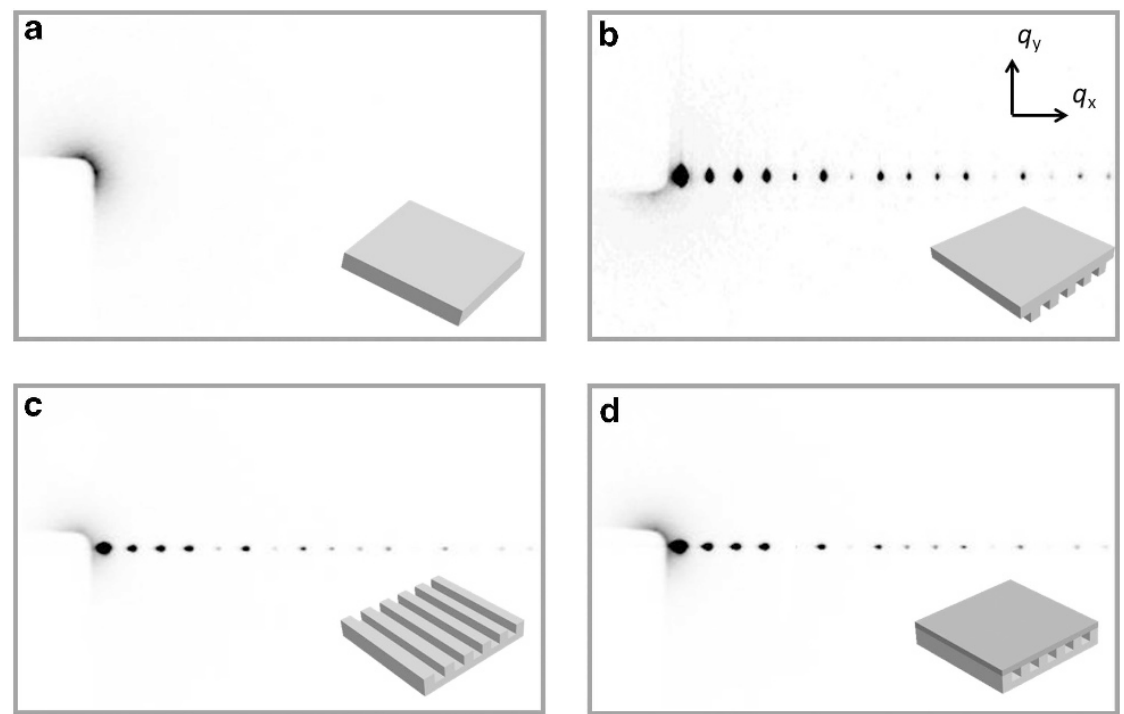

Figure 4 Two-dimensional diffraction patterns obtained from the prepared samples. (a) Compression molded PLA film, (b) the mold with a line pattern, (c) nanoimprinted PLA film (NIL_PLA) and (d) NIL_PLA film covered by a PS thin film (PS/NIL_PLA). A full color version of this figure is available at Polymer Journal online.

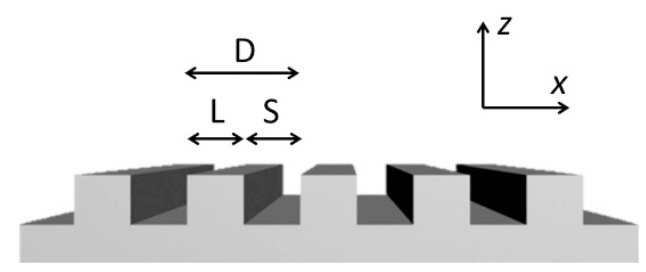

Figure 5 Rectangular LS model of the cross-sectional structure. A full color version of this figure is available at Polymer Journal online.

SR-USAXS could provide accurate information about 'buried' structures and patterns at the outermost surface. Therefore, we can fabricate designed periodic patterns using NIL. Furthermore, we can easily estimate the periodic size of nanoimprinted structure from the peak position. Note that the nanoimprinted structure is useful as a standard sample for determining the beam center and the sample-todetector distance in USAXS.

The line width and the space parts of the nanoimprinted structures were estimated by analyzing the diffraction intensity, assuming that the scatterer was a one-dimensional rectangular LS model, as shown in Figure 5. In this case, the scattering intensity profiles along the $q_{\mathrm{x}}$ direction can be calculated using equation (2):

$$
I\left(q_{x}\right)=A \frac{\sin ^{2}\left(q_{\mathrm{x}} \frac{L}{2}\right)}{q_{\mathrm{x}}^{2}} \frac{\sin ^{2}\left(q_{\mathrm{x}} \frac{\mathrm{ND}}{2}\right)}{\sin ^{2}\left(q_{\mathrm{x}} \frac{D}{2}\right)}
$$

where $A, L, D$ and $N$ are a scaling factor, the line width, the pitch and the number of lattices, respectively.

Figure 6 presents the diffraction intensity plot along the $q_{\mathrm{x}}$ direction from Figure 4 and the corresponding fitted curves calculated using equation (2). Because high-intensity X-rays were used so that higher-order diffraction could be detected, photo-stimulated luminescence saturated the imaging plate ${ }^{19}$ at the first- and second-order diffraction. Therefore, these intensities were not included in the calculation. The diffraction intensity profiles were successfully described by equation (2) using the rectangular LS model. The estimated line width, space width and pitch are listed in Table 2. Here, the fitting parameter $N$ depends on the width of the scattering peaks. However, even when using SR-USAXS to characterize a single NIL_PLA film, the full width at half maximum (FWHM) changed when a different instrumental setup was used. The FWHMs of the sixth-order scattering peak with 4 and $8 \mathrm{~m}$ sample-to-detector distances are $0.004 \mathrm{~nm}^{-1}\left(3.2 \times 10^{-5} \mathrm{rad}\right)$ and $0.003 \mathrm{~nm}^{-1}$ $\left(2.4 \times 10^{-5} \mathrm{rad}\right)$, respectively. The FWHM of the scattering peaks is influenced by sample characteristics, for example, crystallite size and lattice distortion. In addition, the FWHM is broadened by instrumental contributions, as exemplified by light dispersion and the resolution of the detector. This result indicates that the width of the scattering peaks resulting from the sample characteristics is smaller than that arising from the instrumental contributions. Therefore, the characterization of the distortion in the fabricated patterns has limited accuracy. However, the FWHM originating from the NIL_PLA film is $<0.003 \mathrm{~nm}^{-1}$. This value indicates that the distortion in the fabricated patterns is fairly small.

The estimated line width and space parts were $L=241 \mathrm{~nm}$ and $S=172 \mathrm{~nm}$ for the mold and $L=174 \mathrm{~nm}$ and $S=242 \mathrm{~nm}$ for the NIL_PLA film, respectively. The average values and SD of the $L$ and $D$ of each sample with 4 and $8 \mathrm{~m}$ sample-to-detector distances were calculated. For the NIL_PLA film, the average values and SD of $L$ are $174 \mathrm{~nm}$ and $0.93 \mathrm{~nm}$, respectively. The error value is approximately $1 \%$. This value indicates that the instrumental setup does not have a significant influence on the pattern sizes. Here, the nanoimprinted surface pattern on the PLA film is the inverted pattern of the mold. That is, the line part of the mold corresponds to the space part of the nanoimprinted pattern, and vice versa. Therefore, the estimated values were in good agreement between the mold and the NIL_PLA film, indicating that the NIL technique could transfer a surface structure from the mold to the PLA film with high accuracy. In addition, the estimated sizes of the PS/NIL_PLA film were $L=175 \mathrm{~nm}$ and $S=238 \mathrm{~nm}$, which were almost the same as those size of the NIL_PLA film. These results demonstrated that the SR-USAXS technique was highly reliable for the precise and nondestructive characterization of not only the surface structure but also the 'buried' structure. 
$\mathbf{a}$


b
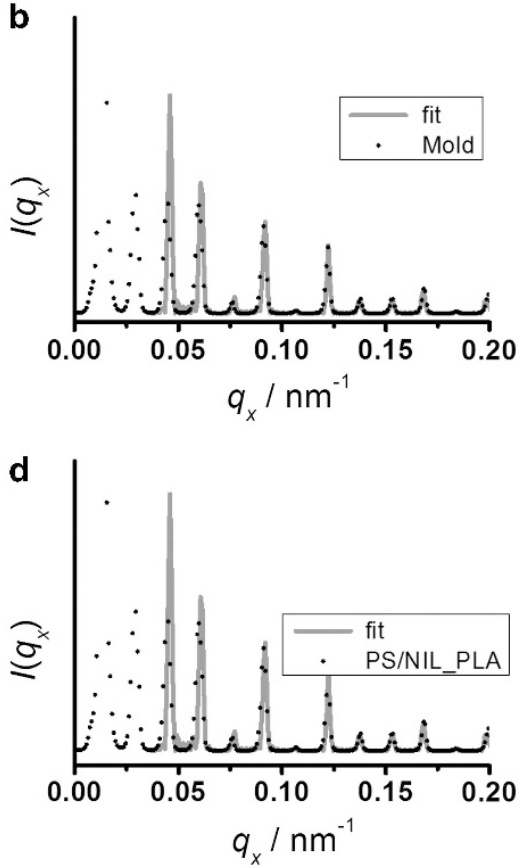

Figure 6 Line-intensity profiles and fitting results of the surface and the 'buried' structure for the PLA films. (a) Compression-molded PLA film, (b) the mold with a line pattern, (c) nanoimprinted PLA film (NIL_PLA) and (d) NIL_PLA film covered by a PS thin film (PS/NIL_PLA). A full color version of this figure is available at Polymer Journal online.

Table 2 Pattern sizes estimated by SR-USAXS measurement

\begin{tabular}{llll}
\hline Sample name & Line $(\mathrm{nm})$ & Space $(\mathrm{nm})$ & Pitch $(\mathrm{nm})$ \\
\hline Mold & $241 \pm 3.2$ & $172 \pm 2.9$ & $413 \pm 2.6$ \\
NIL_PLA & $174 \pm 0.9$ & $242 \pm 3.4$ & $415 \pm 3.4$ \\
PS/NIL_PLA & $175 \pm 1.1$ & $238 \pm 0.6$ & $413 \pm 1.7$ \\
\hline
\end{tabular}

\section{CONCLUSIONS}

In summary, a surface nanostructure was fabricated on PLA films using NIL. To prepare a 'buried' structure, the NIL_PLA film was covered with a PS thin film using the floating method. The fabricated surface and the 'buried' structures were evaluated using SEM measurements and SR-USAXS. The nanostructure on the surface of the PLA film could be observed by SEM. However, the 'buried' structure could not be observed by this method. In contrast, a clear scattering pattern was obtained from the 'buried' structure and the surface structure using SR-USAXS. The sizes of the surface and the 'buried' structure determined from the analysis of the scattering pattern were in good agreement with each other. This study demonstrated that SR-USAXS measurement enables us to evaluate the outermost surface and the 'buried' periodic structure of a nanoimprinted film without any pretreatment steps and without damaging the sample. Furthermore, the nanoimprinted film is a useful standard sample for determining the beam center and the sample-to-detector distance during SAXS.

\section{ACKNOWLEDGEMENTS}

The synchrotron radiation SAXS measurements were performed at SPring-8 with the approval of the Japan Synchrotron Radiation Institute (JASRI, proposal number 2010A7241). The present work is supported by a grant-in-aid for the Global COE Program, 'Science for Future Molecular Systems' and a grant-in-aid for Scientific Research on Innovative Area (20106002) from the MEXT.
1 Kobayashi, M., Yshuda, H. \& Kubodera, K. Japanese patent. 1979022389 (1979).

2 Chou, S. Y., Krauss, P. R. \& Renstrom, P. J. Imprint of sub-25 nm vias and trenches in polymers. Appl. Phys. Lett. 67, 3114-3116 (1995).

3 Honda, K., Morita, M., Masunaga, H., Sasaki, S., Takata, M. \& Takahara, A. Roomtemperature nanoimprint lithography for crystalline poly(fluoroalkyl acrylate) thin films. Soft Matter 6, 870-875 (2010).

4 Honda, K., Morita, M. \& Takahara, A. Room-temperature fabrication of nanotexture in crystalline poly(fluoroalkyl acrylate) thin film. Soft Matter 4, 1400-1402 (2008).

5 Kang, M. G. \& Guo, L. J. Nanoimprinted semitransparent metal electrodes and their application in organic light-emitting diodes. Adv. Mater. 19, 1391-1396 (2007).

6 Li, Y. F., You, S., Zhu, M. W., Zhao, L., Hou, W. T., Detchprohm, T., Taniguchi, Y., Tamura, N., Tanaka, S. \& Wetzel, C. Defect-reduced green GalnN/GaN light-emitting diode on nanopatterned sapphire. Appl. Phys. Lett. 98, 151102 (2011).

7 Han, K.-S., Lee, H., Kim, D. \& Lee, H. Fabrication of anti-reflection structure on protective layer of solar cells by hot-embossing method. Sol. Energy Mater. Sol. Cells 93, 1214-1217 (2009).

8 Atwater, H. A. \& Polman, A. Plasmonics for improved photovoltaic devices. Nat. Mat. 9, 205-213 (2010).

9 Imai, M., Mori, K., Mizukami, T., Kaji, K. \& Kanaya, T. Structural formation of poly(ethylene terephthalate) during the induction period of crystallization: 1. Ordered structure appearing before crystal nucleation. Polymer 33, 4451-4456 (1992).

10 Takahara, A., Hadano, M., Yamaguchi, T., Otsuka, H., Kidoaki, S. \& Matsuda, T. Characterization of novel biodegradable segmented polyurethanes prepared from amino-acid based diisocyanate. Macromol. Symp. 224, 207-218 (2005).

11 Kikuchi, M., Terayama, Y., Ishikawa, T., Hoshino, T., Kobayashi, M., Ogawa, H., Masunaga, H., Koike, J.-i., Horigome, M., Ishihara, K. \& Takahara, A. Chain dimension of polyampholytes in solution and immobilized brush states. Polym. J. 44, 121-130 (2012).

12 Akiba, I., Terada, N., Hashida, S., Sakurai, K., Sato, T., Shiraishi, K., Yokoyama, M., Masunaga, H., Ogawa, H., Ito, K. \& Yagi, N. Encapsulation of a hydrophobic drug into a polymer-micelle core explored with synchrotron SAXS. Langmuir 26, 7544-7551 (2010).

13 Omote, K., Ito, Y. \& Okazaki, Y. A new X-ray metrology for determining cross-sectional profile of semiconductor device pattern. P. Soc. Photo-Opt. Ins. 7638, 763811 (2010).

14 Wu, W. L., Hu, T. J., Jones, R. L., Lin, E. K., Lin, Q. H., Keane, D., Weigand, S. \& Quintana, J. Small angle X-ray scattering metrology for sidewall angle and cross section of nanometer scale line gratings. J. Appl. Phys. 96, 1983-1987 (2004).

15 Ishibashi, Y., Koike, T., Yamazaki, Y., Ito, Y., Okazaki, Y. \& Omote, K. Characterization of cross sectional profile of nanostructure line grating using small angle $x$-ray scattering. P. Soc. Photo-Opt. Ins. 7638, 763812 (2010). 
16 Wu, W. L., Wang, C. Q., Jones, R. L., Lin, E. K., Rice, B. J., Choi, K. W., Thompson, G. Weigand, S. J. \& Keane, D. T. Characterization of correlated line edge roughness of nanoscale line gratings using small angle x-ray scattering. J. Appl. Phys. 102, 024901 (2007).

17 Sakurai, K., Masunaga, H., Ogawa, H., Takano, T., Sasaki, S., Goto, S., Tanaka, T., Seike, T., Takahashi, S., Takeshita, K., Nariyama, N., Ohashi, H., Ohata, T., Furukawa, Y., Matsushita, T., Ishizawa, Y., Yagi, N., Takata, M., Kitamura, H., Tashiro, K., Takahara, A., Amamiya, Y., Horie, K., Takenaka, M., Kanaya, T., Jinnai, H., Okuda, H., Akiba, I., Takahashi, I., Yamamoto, K., Hikosaka, M., Sakurai, S., Shinohara, Y., Okada,
A. \& Sugihara, Y. Multipurpose soft-material SAXS/WAXS/GISAXS beamline at SPring 8. Polym. J. 43, 471-477 (2011).

18 Shinohara, T., Shirahase, T., Murakami, D., Hoshino, T. Kikuchi, M., Koike, J., Horigome, M., Masunaga, H., Ogawa, H. \& Takahara, A. Characterization of surface microstructures on bio-based polymer film fabricated with nano-imprin lithography by synchrotron radiation small angle X-ray scattering. IOP Conf. Ser. 24 012004 (2011)

19 Thoms, M. The dynamic range of X-ray imaging with image plates. Nucl. Instr. Meth Phys. Res. A 389, 437-440 (1997). 